\title{
Transmitter Antenna Diversity and Adaptive Signaling Using Long Range Prediction for Fast Fading DS/CDMA Mobile Radio Channels ${ }^{1}$
}

\author{
Shengquan Hu, Tugay Eyceoz, Alexandra Duel-Hallen \\ North Carolina State University \\ Dept. of Electrical and Computer Engineering \\ Center for Advanced Computing and Communication \\ Box 7914, Raleigh, NC 27695-7914 \\ E-mail: \{shu, teyceoz, sasha\}@eos.ncsu.edu,
}

\author{
Hans Hallen \\ North Carolina State University \\ Physics Department \\ Box 8202, Raleigh, NC 27695-8202 \\ E-mail: Hans_Hallen@ncsu.edu
}

\begin{abstract}
Recently, the authors introduced a novel algorithm for long range prediction of flat fading channels that can reliably predict future fading coefficients far beyond the coherence time. This prediction capability provides enabling technology for power and bandwidth efficient adaptive modulation and coding methods. In this paper, we extend our results to the Direct Sequence Code Division Multiple Access (DS/CDMA) channels. It is demonstrated that frequency selective channels encountered in CDMA can also be predicted far ahead, and that the proposed prediction methods can be combined with RAKE receivers. In addition, we utilize prediction to improve performance of the selective transmitter diversity system proposed for the third generation CDMA.
\end{abstract}

\section{INTRODUCTION}

DS/CDMA technology is attractive for wireless access because of its numerous advantages over other multiple access techniques. The Wideband DS/CDMA (WCDMA) has been developed as a predominant radio access technology for the next generation global wireless standard [1,2]. One of the novel features of WCDMA is support for transmitter antenna diversity for the downlink $[3,4,5]$.

Some of the proposed transmitter antenna diversity schemes are Orthogonal Transmitter Diversity (OTD), Transmitter Adaptive Array (TxAA), Selective Transmitter Diversity (STD), and Space Time Transmitter Diversity (STTD). TxAA and STD can result in better performance than OTD and STTD, but require feedback of channel state information (CSI) from the mobile to the base station. In practice, the performance of these schemes can be degraded due to imperfect and delayed CSI. In particular, even small delay can result in significant degradation due to the time varying nature of the fading channel. In this paper, we propose to utilize the long range prediction method [6-9] to improve the performance of transmitter diversity systems. In particular, we focus on Selective Transmitter Diversity. Accurate prior knowledge of the channel for the entire duration of the next frame or slot provided by long-term prediction would enable more efficient antenna switching at the transmitter.

In the next section, we review the CDMA channel and analyze an ideal STD+RAKE system. In Section 3, the long range prediction method is summarized. A comparison of the mean square errors for three approaches to long range total power prediction for multipath channels (required for STD implementation) is carried out in Section 4. In Section 5, the Bit Error Rate (BER) gain achieved by long term prediction for an STD+RAKE system is demonstrated.

\section{CDMA SYSTEM MODEL AND STD}

The WCDMA channel is often frequency-selective due to large transmission bandwidth $[1,2,10]$. As a result, large diversity gain can be realized using the RAKE receiver. This gain can be further enhanced by utilized antenna (diversity) arrays. In practice, the mobile is often limited to a single antenna, whereas the base station can employ several antennas. Thus, transmitter antenna diversity techniques for down link signaling have been recently investigated by many researchers (e.g., [3-5].) We consider a combined Selective Transmitter Diversity and RAKE receiver (STD+RAKE) system shown in Figure 1 for two antennas (it can be easily extended to a greater number of antennas). When the signal of the desired user is transmitted either from antenna $\mathrm{A}$ or from antenna $\mathrm{B}$, the channel is characterized as frequencyselective Rayleigh fading with $\mathrm{L}$ paths. All paths are i.i.d. and each channel has unit average power, i.e., $\mathrm{E}\left\{\left|\mathrm{c}_{\mathrm{k}}{ }^{\mathrm{A}}\right|^{2}\right\}=$ $\mathrm{E}\left\{\left|\mathrm{c}_{\mathrm{k}}^{\mathrm{B}}\right|^{2}\right\}=1 / \mathrm{L}, \mathrm{k}=1, \cdots, \mathrm{L}$. The total instantaneous powers associated with the channels of antennas $\mathrm{A}$ and $\mathrm{B}$ are: $\Omega^{\mathrm{A}}=$ $\sum_{\mathrm{k}=1}^{\mathrm{L}}\left|\mathrm{c}_{\mathrm{k}}{ }^{\mathrm{A}}\right|^{2}$, and $\Omega^{\mathrm{B}}=\sum_{\mathrm{k}=1}^{\mathrm{L}}\left|\mathrm{c}_{\mathrm{k}}{ }^{\mathrm{B}}\right|^{2}$, respectively. Both $\Omega^{\mathrm{A}}$ and $\Omega^{\mathrm{B}}$ are Chi-Square distributed with $2 \mathrm{~L}$ degrees of freedom. For an STD system, the transmission antenna is selected based on the power comparison between $\Omega^{\mathrm{A}}$ and $\Omega^{\mathrm{B}}$. The antenna that results in a stronger signal at the receiver will be used as the transmission antenna. Thus, assuming perfect CSI at the transmitter, the channel power of the STD system is $\Omega^{\mathrm{S}}$, and the selected multipath fading channel components are $\left\{\mathrm{c}_{1}{ }^{\mathrm{s}}, \mathrm{c}_{2}{ }^{\mathrm{s}}, \cdots, \mathrm{c}_{\mathrm{L}}{ }^{\mathrm{s}}\right\}$, where $\mathrm{s}=\underset{\mathrm{A} \text { or B }}{\operatorname{argmax}}\left\{\Omega^{\mathrm{A}}, \Omega^{\mathrm{B}}\right\}$. The probability density function (pdf) of $\Omega^{\mathrm{S}}$ is:

$$
\mathrm{f}_{\Omega} \mathrm{s}(\mathrm{x})=\frac{2 \mathrm{~L}^{\mathrm{L}}}{(\mathrm{L}-1) !} \mathrm{x}^{\mathrm{L}-1} \mathrm{e}^{-\mathrm{Lx}}\left(1-\mathrm{e}^{-\mathrm{Lx}} \sum_{\mathrm{k}=0}^{\mathrm{L}-1}(\mathrm{Lx})^{\mathrm{k}} \frac{1}{\mathrm{k} !}\right)
$$

Thus, the received signal of the desired user is modeled as

$$
r(t)=\sum_{k=0}^{\infty} \sum_{i=1}^{L} c_{i}^{s}(t) s\left(t-k T-i T_{c}\right) b_{k}+z(t)
$$

where $b_{k}$ is the binary phase shift keying (BPSK) data sequence, $\mathrm{T}$ is the symbol interval, $\mathrm{s}(\mathrm{t})$ is the signature sequence, $T_{c}$ is the chip duration, and $z(t)$ is a complexvalued zero-mean white Gaussian noise process with variance

\footnotetext{
${ }^{1}$ This research was supported by NSF grants CCR-9725271 and NCR-9726033
} 


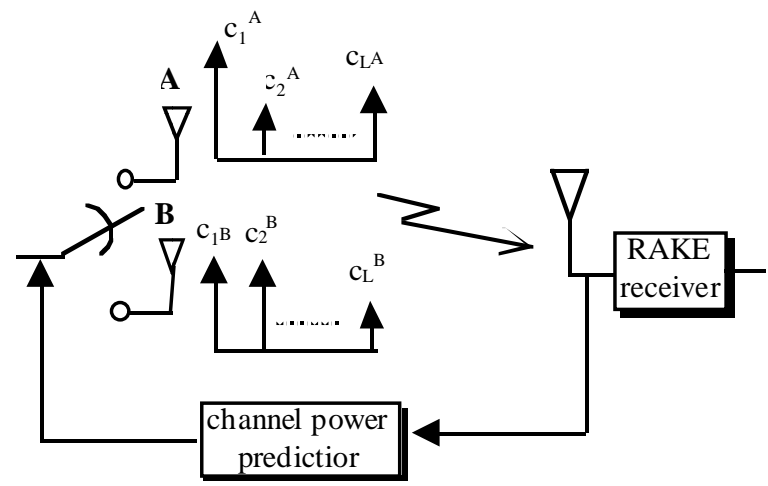

Figure 1. Configuration of combined STD + RAKE system

$\mathrm{N}_{0} / 2$. The noise term in this paper models multiuser and intersymbol interference and thermal noise. We also assume that the L multipath-delayed versions of the signature waveform $s(t)$ are orthogonal to each other. The RAKE receiver with maximal ratio combining collects the powers of the $\mathrm{L}$ multipath components at the receiver [10]. The instantaneous SNR per bit is given by:

$$
\gamma_{\mathrm{b}}{ }^{\mathrm{s}}=\frac{\mathrm{E}_{\mathrm{b}}}{\mathrm{N}_{0}} \sum_{\mathrm{k}=1}^{\mathrm{L}}\left|\mathrm{c}_{\mathrm{k}}^{\mathrm{s}}\right|^{2}=\mathrm{L} \gamma_{\mathrm{c}} \Omega^{\mathrm{s}}
$$

where $E_{b}$ is the transmitted energy per bit, $\gamma_{c}=\frac{E_{b}}{L_{0}}$. The pdf of $\gamma_{b}{ }^{\mathrm{s}}, f \gamma_{b}{ }^{\mathrm{s}}(\mathrm{x})$, can be determined from (1) and (3). Then the BER of the STD+RAKE system can be obtained as:

$$
\mathrm{P}_{\mathrm{b}}=\int_{0}^{\infty} \mathrm{Q}(\sqrt{2 \mathrm{x}}) \mathrm{f} \gamma_{\mathrm{b}}^{\mathrm{s}}(\mathrm{x}) \mathrm{dx}
$$

For $\mathrm{L}=2$, the closed form of $\mathrm{P}_{\mathrm{b}}$ is:

$$
\begin{gathered}
\mathrm{P}_{\mathrm{b}}=2(2+\mu)\left(\frac{1-\mu}{2}\right)^{2}-\frac{1}{2}\left(2+\mu^{\prime}\right)\left(\frac{1-\mu}{2}\right)^{2}- \\
\frac{1}{2}\left(\left(\frac{1-\mu^{\prime}}{2}\right)^{3}\left(1+\frac{3}{2}\left(1+\mu^{\prime}\right)+\frac{3}{2}\left(1+\mu^{\prime}\right) 2\right)\right)
\end{gathered}
$$$$
\text { where } \mu=\sqrt{\frac{\gamma_{c}}{1+\gamma_{c}}} \text {, and } \mu^{\prime}=\sqrt{\frac{\gamma_{c}}{2+\gamma_{c}}} \text {. }
$$

Eq. (5) is the lower bound on the BER of STD+RAKE, since the derivation above assumes perfect CSI at the transmitter and the receiver. In practice, the propagation delay, fading channel variation, feedback channel errors and receiver channel estimation errors degrade CSI. In the following two sections, we discuss the utilization of the long term prediction in overcoming CSI inaccuracies at the transmitter associated with the propagation delay and fading.

\section{Long Range Prediction of the Fading Channel}

The long range prediction capability for the complex valued fading channel was demonstrated in [6-9] and references therein. In previous work, we concentrated on flat fading signals. Our linear prediction (LP) method is based on the AR channel modeling. Assume that the equivalent complex Rayleigh fading process $c(t)$ is sampled at the rate $f_{s}$ $=1 / T_{\mathrm{s}}$, where $\mathrm{f}_{\mathrm{s}}$ is at least twice the maximum Doppler shift, $f_{d m}[11]$. Let $c_{j}=c\left(j T_{s}\right)$. The linear MMSE prediction of the future channel sample $\hat{c}_{n}$ based on p previously observed channel samples $\mathrm{c}_{\mathrm{n}-1}, \mathrm{c}_{\mathrm{n}-2}, \ldots, \mathrm{c}_{\mathrm{n}-\mathrm{p}}$ is

$$
\hat{c}_{n}=\sum_{j=1}^{p} d_{j} c_{n-j}
$$

where $d_{j}$ 's are the coefficients of the LP filter. The optimal coefficients $\mathrm{d}_{\mathrm{j}}$ 's are computed as

$$
\underline{\mathrm{d}}=\underline{\mathrm{R}}^{-1} \underline{\mathrm{r}}
$$

where $\underline{d}=\left(d_{1} \cdots d_{p}\right) . \underline{R}$ is the autocorrelation matrix $(p \times p)$ with coefficients $R_{i j}=E\left[c_{n-i} c_{n-j}^{*}\right]$ and $\underline{r}$ is the autocorrelation vector $(p \times 1)$ with coefficients $r_{j}=E\left[c_{n} c^{*}{ }_{n+j}\right]$. Therefore, the resulting MMSE is given by

$$
E\left[\left|e_{n}\right|^{2}\right]=E\left[\left|c_{n}-\hat{c}_{n}\right|^{2}\right]=r_{0}-\sum_{j=1}^{p} d_{j} r_{j}
$$

The LP can be generalized to predict any time $\tau$ ahead, where $\tau$ is the prediction range [7]. In (7), the prediction is one step ahead, i.e. $\tau=\mathrm{T}_{\mathrm{s}}$. In practical implementation, we iterate this one-step prediction to forecast the channel further than one sampling interval ahead by utilizing previously predicted samples instead of the observations. It was shown in $[6,7]$ that the key to the long range prediction is the sufficiently long memory span achieved for moderate model order $p$ by selecting low sampling rate, $f_{s}$ (much lower than the data rate). Interpolation is used to perform prediction at the data rate. Furthermore, an adaptive decision-directed longrange prediction method allows to reduce the effect of noise and to keep up with the channel variations. In addition to the theoretical analysis and simulations for the Rayleigh fading and the Jakes model [11], we verified the performance of long range prediction for realistic physical channel models and measured data $[8,9]$.

Our previous investigations of the long range prediction for the fading channel focused on the case when the complex valued flat-fading coefficients were predicted and observed. However, depending on the application, a different prediction problem might be of interest. For example, in the decision directed channel estimation, phase ambiguity requires differential encoding, and absolute phases are not available. This makes prediction of future phases problematic. But this is not a serious limitation, since implementation of many proposed adaptive coding methods, e.g. [12-14], depends on the knowledge of future power only, and phase prediction is not necessary. Thus, it is desirable to examine long range prediction of the fading channel power using observed power samples (see also [8]).

In the STD+RAKE systems for WCDMA, it is of interest to determine the future total power for each antenna to aid the antenna selection. However, in contrast to the channels with phase ambiguity, in the proposed $3^{\text {rd }}$ generation WCDMA systems, coherent channel estimates are available at the receiver since the pilot channel is used. Therefore, complex fading coefficients associated with different multipath components and transmitter antennas (see Section 2) can serve as observations. As we show below, several approaches to future power prediction can be exploited depending on the performance/complexity requirements. 


\section{STD WITH PREDICTION IN WCDMA SYSTEMS}

One of the key features that makes WCDMA feasible globally is its high carrier frequency of $2 \mathrm{GHz}$. However, this high carrier frequency results in very large Doppler shifts at moderate vehicular speeds (e.g. $65 \mathrm{mi} / \mathrm{h}$ corresponds to $\mathrm{f}_{\mathrm{dm}}=$ $200 \mathrm{~Hz}$.) These high Doppler shifts result in significant variations of the fading channel coefficient over short time periods. Thus, outdated channel estimates fed back to the transmitter become less useful for adaptive signaling application, and long-term fading prediction capability becomes more important. Using accurately predicted future channel power, the transmitter can appropriately select the signaling method for the future frame even when channel varies rapidly due to fast fading.

In this section, we analyze three approaches to long-range prediction of the down link channel power for each transmitter antenna given a sequence of channel observations associated with that antenna. This information about future channel power allows the mobile to make a suitable selection of the base station antenna for the next transmission interval. In this analysis, we assume that present and past samples of the i.i.d. Rayleigh fading coefficients $c_{k}$ for $L$ paths $(k=1, \ldots, L)$ are observed at the mobile for each transmitter antenna (i.e. samples of $\mathrm{c}_{\mathrm{k}}{ }^{\mathrm{A}}(\mathrm{t})$ and $\mathrm{c}_{\mathrm{k}}{ }^{\mathrm{B}}(\mathrm{t})$ as in section 2 are observed, but we suppress the antenna (A or $\mathrm{B}$ ) and time indices in the sequel). This analysis can be extended to include noise present in the observations (e.g. noisy pilot symbols). In this paper, we restrict the derivation to the noiseless case to show the potential of long-term power prediction for the ideal Rayleigh fading channel, and the noisy case is examined through simulation. In the derivations, (7-9) are generalized to include different observation processes and arbitrary prediction range $\tau$.

Case 1: In this approach, each future complex Gaussian fading coefficient $c_{k}(t)$ is predicted separately for each path and each antenna as in (7), and the total predicted power for each antenna is calculated using these estimates. These future predicted samples are denoted as $\hat{c}_{1}, \hat{c}_{2}, \ldots, \hat{c}_{L}$. The autocorrelation function of each component is [15]:

$$
\mathrm{r}_{\mathrm{j}}=(1 / \mathrm{L})\left(\Omega_{0} / 2\right) \mathrm{J}_{0}\left(2 \pi \mathrm{f}_{\mathrm{dm}} \mathrm{j} \mathrm{T}_{\mathrm{s}}\right)
$$

where $\mathrm{E}\left[\left|\mathrm{c}_{\mathrm{i}}\right|^{2}\right]=\Omega_{0} / \mathrm{L}$. The prediction MMSE per component is $\xi_{\mathrm{i}}=\mathrm{E}\left[\left|\mathrm{e}_{\mathrm{i}}\right|^{2}\right]$, where $\mathrm{e}_{\mathrm{i}}=\mathrm{c}_{\mathrm{i}}-\hat{\mathrm{c}}_{\mathrm{i}}$. By the orthogonality principle,

$$
\mathrm{E}\left[\left|\hat{\mathrm{c}}_{\mathrm{i}}\right|^{2}\right]=\mathrm{E}\left[\mathrm{c}_{\mathrm{i}} \hat{\mathrm{c}}_{\mathrm{i}}^{*}\right]=\mathrm{E}\left[\mathrm{c}_{\mathrm{i}}{ }^{*} \mathrm{c}_{\mathrm{i}}\right]=\mathrm{E}\left[\left|\mathrm{c}_{\mathrm{i}}\right|^{2}\right]-\xi_{\mathrm{i}}
$$

Since each $\mathrm{c}_{\mathrm{i}}$ has complex Gaussian distribution, the estimate $\hat{\mathrm{c}}_{\mathrm{i}}$ and the error $\mathrm{e}_{\mathrm{i}}$ are also complex Gaussian (see (7)). Thus,

$$
\mathrm{E}\left[\left|\hat{\mathrm{c}}_{\mathrm{i}}\right|^{4}\right]=2\left(\mathrm{E}\left[\left|\mathrm{c}_{\mathrm{i}}\right|^{2}\right]-\xi_{\mathrm{i}}\right)^{2}=2\left(\Omega_{0} / \mathrm{L}-\xi_{\mathrm{i}}\right)^{2}
$$

The total power prediction mean squared error is

$$
\xi_{\mathrm{T}}=\mathrm{E}\left[\left|\sum_{\mathrm{i}=1}^{\mathrm{L}}\left(\left|\mathrm{c}_{\mathrm{i}}\right|^{2}-\left|\hat{\mathrm{c}}_{\mathrm{i}}\right|^{2}\right)\right|^{2}\right]=\sum_{\mathrm{i}=1}^{\mathrm{L}} \mathrm{E}\left[\left|\mathrm{e}_{\mathrm{i}}\right|^{2}\right]+\sum_{\mathrm{k} \neq \mathrm{j}} \mathrm{E}\left[\mathrm{e}_{\mathrm{k}}{ }^{\prime} \mathrm{e}_{\mathrm{j}}{ }^{\prime}\right]
$$

where $\mathrm{e}_{\mathrm{i}}^{\prime}=\left|\mathrm{c}_{\mathrm{i}}\right|^{2}-\left|\hat{\mathrm{c}}_{\mathrm{i}}\right|^{2}$. Express

$$
\xi_{i}^{\prime}=E\left[\left|e_{i}^{\prime}\right|^{2}\right]=E\left[\left|\mathrm{c}_{i}\right|^{4}\right]+E\left[\left|\hat{c}_{i}\right|^{4}\right]-2 E\left[\left|\mathrm{c}_{i}\right|^{2}\left|\hat{c}_{\mathrm{i}}\right|^{2}\right]
$$

where $\mathrm{E}\left[\left|\mathrm{c}_{\mathrm{i}}\right|^{2}\left|\hat{\mathrm{c}}_{\mathrm{i}}\right|^{2}\right]=\mathrm{E}\left[\left|\mathrm{c}_{\mathrm{i}}\right|^{4}\right]-\xi_{\mathrm{i}}{ }^{\prime}$. Thus,

$$
\xi_{\mathrm{i}}^{\prime}=\mathrm{E}\left[\left|\mathrm{c}_{\mathrm{i}}\right|^{4}\right]-\mathrm{E}\left[\left|\hat{\mathrm{c}}_{\mathrm{i}}\right|^{4}\right]=4\left(\Omega_{0} / \mathrm{L}\right) \xi_{\mathrm{i}}-2 \xi_{\mathrm{i}}^{2}
$$

Using (15), the fact that $\mathrm{e}_{\mathrm{k}}{ }^{\prime}$ are i.i.d., and $\mathrm{E}\left[\mathrm{e}_{\mathrm{k}}{ }^{\prime}\right]=\xi_{\mathrm{i}}$, we find the total mean squared error for Case 1 as:

$$
\xi_{\mathrm{T}}=4 \Omega_{0} \xi_{\mathrm{i}}+\left(\mathrm{L}^{2}-3 \mathrm{~L}\right) \xi_{\mathrm{i}}^{2}
$$

Since $\xi_{\mathrm{i}}=\xi_{\text {flat }} / \mathrm{L}$, where $\xi_{\text {flat }}$ is the MMSE of the complex fading coefficient prediction for $\mathrm{L}=1$, the $\xi_{\mathrm{T}}$ is given as

$$
\xi_{\mathrm{T}}=\left(4 \Omega_{0} / \mathrm{L}\right) \xi_{\text {flat }}+\left(\mathrm{L}^{2}-3 \mathrm{~L}\right) / \mathrm{L}^{2} \xi_{\text {flat }}^{2}
$$

Case 2: In this case, we apply linear MMSE prediction directly to the observations of powers $\Omega_{1,}, \Omega_{2,}, \ldots, \Omega_{\mathrm{L}}$, where $\Omega_{\mathrm{i}}=\left|\mathrm{c}_{\mathrm{i}}\right|^{2}$ represents the power of the fading channel associated with the i-th multipath component for a given antenna. The total predicted power will be computed using these individual estimates $\hat{\Omega}_{\mathrm{i}}$. This Case (and Case 3 ) are particularly useful to investigate for channels with phase ambiguity since they do not require the knowledge of the phases of the fading coefficients. Each $\Omega_{\mathrm{i}}$ has the autocorrelation function [15]:

$$
\mathrm{r}(\tau)=\left(\Omega_{0} / \mathrm{L}\right)^{2} \mathrm{~J}_{0}^{2}\left(2 \pi \mathrm{f}_{\mathrm{dm}} \tau\right)+\left(\Omega_{0} / \mathrm{L}\right)^{2}
$$

The total prediction MSE is $\xi_{\mathrm{T}}=\mathrm{E}\left[\left|\sum_{\mathrm{i}=1}^{\mathrm{L}}\left(\Omega_{\mathrm{i}}-\hat{\Omega}_{\mathrm{I}}\right)\right|^{2}\right]$. Similarly to Case 1 , the total MSE can be expressed as

$$
\xi_{\mathrm{T}}=\mathrm{L} \xi_{\mathrm{i}}+\mathrm{L}(\mathrm{L}-1)\left(\Omega_{0} / \mathrm{L}\right)^{2}\left(1-\sum_{\mathrm{j}=1}^{\mathrm{p}} \mathrm{d}_{\mathrm{j}}\right)^{2}
$$

where $\xi_{\mathrm{i}}=\mathrm{E}\left[\left|\Omega_{\mathrm{i}}-\hat{\Omega}_{\mathrm{i}}\right|^{2}\right]$, and $\mathrm{d}_{\mathrm{j}}$ are the LP coefficients for the MMSE power prediction calculated as in (8) using the autocorrelation function $\mathrm{r}(\tau)$ (18). Also, from (18), $\xi_{\mathrm{i}}=$ $\left(\Omega_{0} / \mathrm{L}\right)^{2}\left(1-\sum_{\mathrm{j}=1}^{\mathrm{p}} \mathrm{d}_{\mathrm{j}}\right)+\left(\Omega_{0} / \mathrm{L}\right)^{2}\left(1-\sum_{\mathrm{j}=1}^{\mathrm{p}} \mathrm{d}_{\mathrm{j}} \mathrm{J}_{0}{ }^{2}\left(2 \pi \mathrm{f}_{\mathrm{dm}} \mathrm{j} \mathrm{Ts}\right)\right)$. Therefore the total MSE for Case 2 is given as:

$$
\begin{gathered}
\xi_{\mathrm{T}}=\Omega_{0}{ }^{2}\left(1-\sum_{\mathrm{j}=1}^{\mathrm{p}} \mathrm{d}_{\mathrm{j}}\right)^{2}+\left(\Omega_{0}{ }^{2} / \mathrm{L}\right)\left(1-\sum_{\mathrm{j}=1}^{\mathrm{p}} \mathrm{d}_{\mathrm{j}}\right)\left(\sum_{\mathrm{j}=1}^{\mathrm{p}} \mathrm{d}_{\mathrm{j}}\right)+ \\
\Omega_{0}{ }^{2} / \mathrm{L}\left(1-\sum_{\mathrm{j}=1}^{\mathrm{p}} \mathrm{d}_{\mathrm{j}} \mathrm{J}_{0}{ }^{2}\left(2 \pi \mathrm{f}_{\mathrm{dm}} \mathrm{j} T \mathrm{~s}\right)\right)
\end{gathered}
$$

Case 3: In this approach, we form the linear MMSE prediction of the total power of the fading channel for each antenna using previous total power samples observed at the receiver. The total power is given as $\Omega_{\mathrm{T}}=\Omega_{1}+\Omega_{2}+\ldots+$ $\Omega_{\mathrm{L}}$. It can be shown that the autocorrelation function of $\Omega_{\mathrm{T}}$ is

$$
\mathrm{r}_{\mathrm{T}}(\tau)=\left(\Omega_{0} / \mathrm{L}\right)^{2} \mathrm{~J}_{0}^{2}\left(2 \pi \mathrm{f}_{\mathrm{dm}} \tau\right)+\Omega_{0}^{2},
$$

and the MMSE E[| $\left.\Omega_{\mathrm{T}}-\left.\hat{\Omega}_{\mathrm{T}}\right|^{2}\right]$ for Case 3 can be expressed as

$$
\xi_{\mathrm{T}}=\Omega_{0}{ }^{2}\left(1-\sum_{\mathrm{j}=1}^{\mathrm{p}} \mathrm{d}_{\mathrm{j}}\right)+\Omega_{0}{ }^{2} / \mathrm{L}\left(1-\sum_{\mathrm{j}=1}^{\mathrm{p}} \mathrm{d}_{\mathrm{j}} \mathrm{J}_{0}{ }^{2}\left(2 \pi \mathrm{f}_{\mathrm{dm}} \mathrm{j} \mathrm{Ts}\right)\right)
$$

Performance Comparisons of 3 Cases: We compare the MSEs of the three approaches derived above for the noiseless case. In our analysis, the total channel power for each antenna is normalized to 1 . To make system parameters consistent with the third-generation WCDMA system, we assume the carrier frequency is $2 \mathrm{GHz}$ and $\mathrm{f}_{\mathrm{dm}}=200 \mathrm{~Hz}$. In the theoretical calculation of the model coefficients, it is required to invert the autocorrelation matrices obtained from sampling (10), (18) and (21) for Cases 1, 2, and 3, respectively. We found that higher sampling rates $\mathrm{f}_{\mathrm{s}}$ (e.g., $1.6 \mathrm{KHz}$ ) cause matrix singularities when the model $\mathrm{p}$ is large. This is due to oversampling the channel relative to the Nyquist rate of $400 \mathrm{~Hz}$. If the sampling rate is chosen closer to $400 \mathrm{~Hz}$, the 


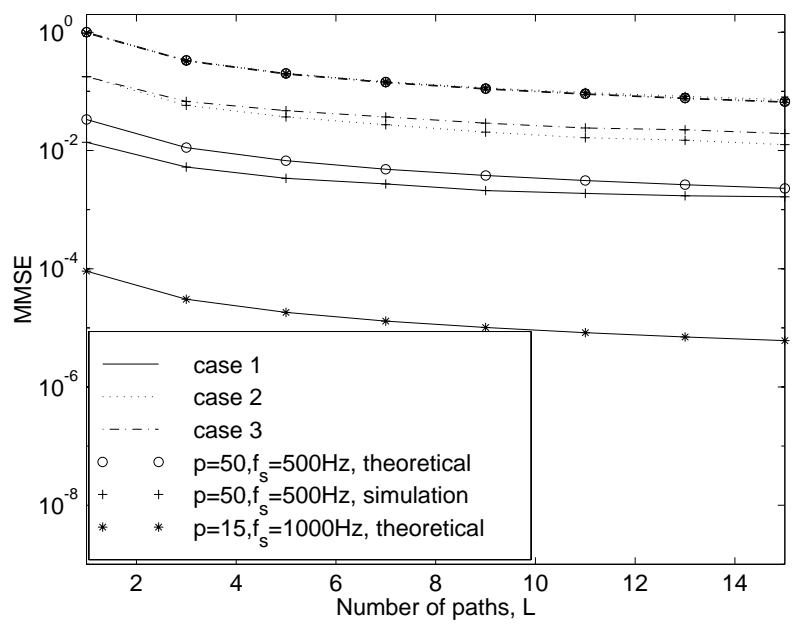

Figure 2. Prediction MSE of total multipath channel power. Prediction range $\tau=2 \mathrm{~ms}$.

matrix does not become singular for large p. In practice, additive noise and the finite observation interval result in a non-singular matrix. In this comparison of the ideal noiseless MSEs, we concentrate on cases when the matrix is not singular. This is assured by choosing sufficiently low $f_{s}$ for a given value of $\mathrm{p}$.

In Figure 2, we fix the prediction range $\tau=2 \mathrm{~ms}$, and examine two choices of prediction parameters. The first selection $\left(p=50, f_{s}=500 \mathrm{~Hz}\right)$ corresponds to much larger memory span $\mathrm{p} / \mathrm{f}_{\mathrm{s}}$ than the second set $\left(\mathrm{p}=15, \mathrm{f}_{\mathrm{s}}=1 \mathrm{KHz}\right)$. Nevertheless, the second selection results in much lower MSE for Case 1, suggesting that it is beneficial to sample recent observation at sufficiently high rate (of course $1 \mathrm{KHz}$ is still much lower than the data rate). We find that the total MSEs for all 3 cases decrease as L grows and approach the saturation values that can be determined from (17), (20), and (22). The theoretical MSEs of cases 2 and 3 for both choices are close enough to be considered as same. In general, these cases perform much poorer than Case 1 for realistic prediction ranges and number of paths. However, Case 3 requires only one predictor per antenna, so its complexity is lower than that of Cases 1 and 2, which require L predictors for each antenna. Of course, in the presence of phase ambiguity Case 1 is not feasible, and Case 3 is the better choice. In Figure 2, we also show simulation results for the noiseless case for the lower $\mathrm{f}_{\mathrm{s}}$. They were implemented using 2000 observation samples for the Jakes model with 100 oscillators. Due to the channel mismatch [7], simulations do not closely match theoretical results, but performance trends are the same.

In Figure 3, we consider the MSE performance versus the prediction range for the sampling rate, $\mathrm{f}_{\mathrm{s}}=1.6 \mathrm{KHz}$, and $p=10$. Observe that Cases 2 and 3 outperform Case 1 for sufficiently large prediction range. We also found that as $\mathrm{L}$ increases and $\mathrm{f}_{\mathrm{s}}$ decreases, this cross-over occurs for a lower value of the prediction range. Thus, when prediction far ahead is desired, Case 1 is not always the best choice, considering its high complexity. However, for most practical applications Case 1 would result in the best performance.

We also examined performance of our prediction method with noisy observations by simulation (not shown). It was

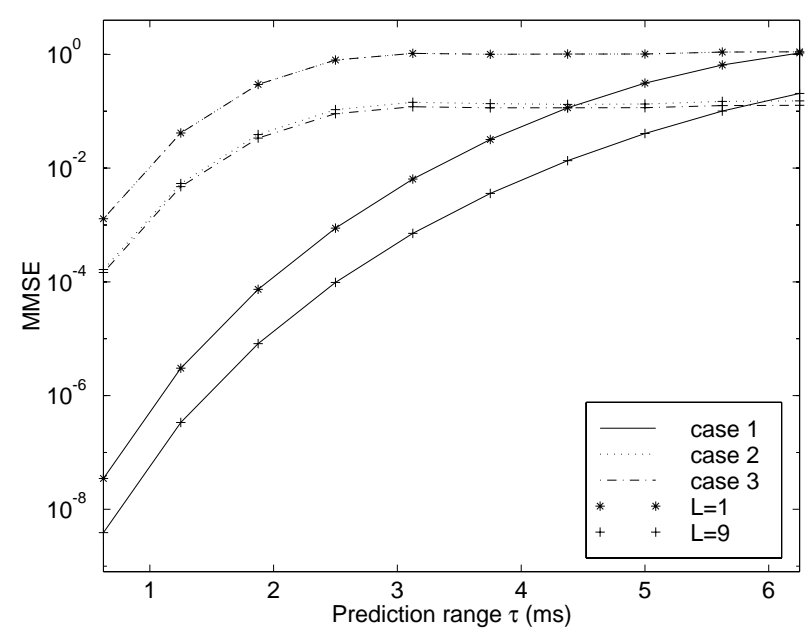

Figure 3. Theoretical prediction MSE vs. prediction range.

found that MSE performance degrades greatly for low SNR (all three cases perform above MSE of $10^{-1}$ for $\mathrm{SNR}=10 \mathrm{~dB}$ ). Therefore, it is important to reduce the effect of noise. This can be accomplished in practice by combining prediction with adaptive tracking [7-9].

\section{BIT ERROR RATE OF STD+RAKE}

We present the BER simulation results of the long range channel prediction algorithm for STD+RAKE system shown in Figure 1. Jakes model with nine oscillators was used to generate the fading channels. We sample the channel at the rate of $1.6 \mathrm{kHz}$, which corresponds to the power control rate in the WCDMA system [1]. Assume the transmission data rate is $80 \mathrm{kbps}$ and $\mathrm{f}_{\mathrm{dm}}=200 \mathrm{~Hz}$.

First, for a flat fading channel, we investigate STD with different switching frequencies corresponding to one slot $(1.6 \mathrm{KHz})$, four slots $(400 \mathrm{~Hz})$ and one frame $(160 \mathrm{~Hz})$ of the WCDMA system. Assume that antenna selection bit is chosen based on the power comparison at the receiver and sent to the transmitter. This selection is made during the slot that precedes the antenna switching. When no prediction is performed, the outdated channel estimates are used to make this selection, since fading conditions during previous slot determine which antenna is going to be chosen. This feedback delay is given by the duration of one slot.

When prediction is utilized (we assume noiseless observations and use Case 1), previous channel samples collected at the rate of $1.6 \mathrm{KHz}$ are used to predict the channel power during the next switching interval. The most recent sample is delayed by $0.625 \mathrm{~ms}$ with respect to the beginning of the switching interval - same delay as when prediction is not employed. Antenna switching is based on the average of interpolated channel samples at the data rate. These interpolated data rate samples are computed using predicted lower rate $(1.6 \mathrm{KHz})$ channel samples. Multi-step prediction is performed to forecast sufficient number of samples. To reduce complexity, we can avoid interpolation by averaging the points predicted at the lower sampling rate without significantly degrading the BER performance. The prediction technique employed in this paper does not utilize adaptive tracking. Adaptation would further improve prediction 


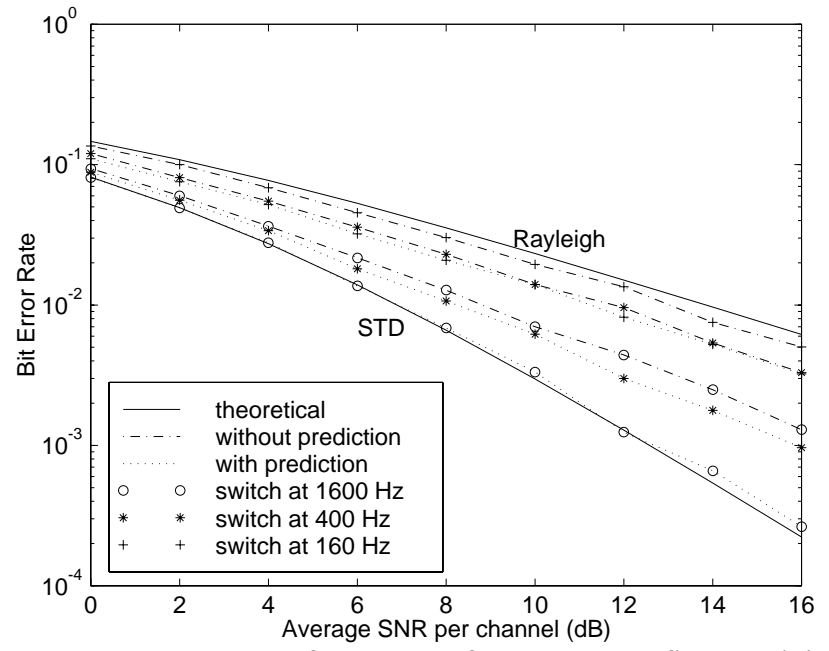

Figure 4. BER performance of STD over flat Rayleigh fading channel with and without prediction for various antenna switching rates.

accuracy by reducing the effect of the finite observation interval.

Figure 4 also contains the BER of the Rayleigh fading channel [ 10] and the ideal performance of the STD (5). We conclude that significant performance gains ( $2 \mathrm{~dB}$ or greater) are achieved for fast vehicle speeds when long range prediction is used.

Now, we consider the BER performance of STD+RAKE system shown in Figure 1 for $\mathrm{L}=2$. Theoretical BER performance is given by (5). In the simulation, we adaptively select the antenna at the rate of $1.6 \mathrm{KHz}$. The BERs of STD+RAKE system obtained using simulations with three different power prediction approaches proposed in Section 3 are plotted in Figure 5. As expected, Case 1 gives the best performance. However, the gain with respect Case 2 and 3 is not very significant. This is because STD is not as sensitive to the prediction error as adaptive power control schemes (e.g. truncated channel inversion [6]), since only the information on the ranking of channel powers is required. Finally, the BER of STD with 1-slot delayed channel state information (without prediction) was also investigated by simulations. As in the flat fading case, our power prediction method achieves significant performance gains over the delayed channel estimation.

As the number of paths increases, the ideal performance of STD+RAKE given by (5) improves, although saturation occurs for large L. Long range prediction helps to realize the gains for this promising downlink diversity technique for rapidly varying multipath fading channels.

\section{Conclusion}

We evaluated performance of the transmitter selection diversity technique for multipath fading CDMA channels aided by long range power prediction algorithms. Suitable choice of prediction parameters results in accurate prediction that makes STD feasible even for high vehicle speeds.

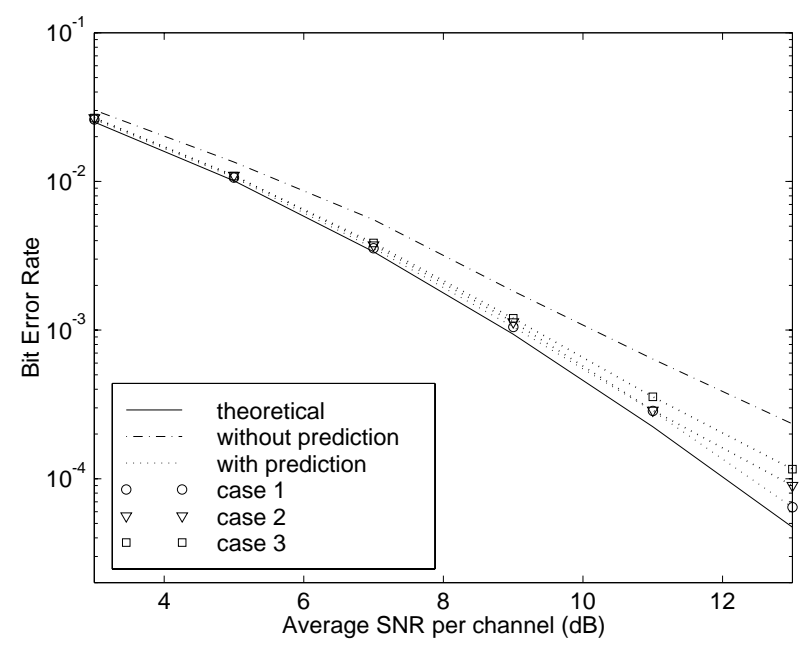

Figure 5. BER performance comparison of STD+RAKE (switching frequency $1.6 \mathrm{KHz}, \mathrm{L}=2$ ) without prediction and for three power prediction methods.

\section{ACKNOWLEDGMENT}

The authors would like to thank Secin Guncavdi and Dr. Dimitrios Efstathiou for helpful discussions.

\section{REFERENCES}

[1] E. Dahlman et al., "WCDMA - The Radio interface for Future Mobile Multimedia Communications", IEEE Trans. on Vehicular Tech.., vol. 47, No. 4, pp. $1105-1117$, Nov. 1998.

[2] F. Adachi, M. Sawahashi, and H. Suda, "Wideband DS-CDMA for Next Generation Mobile Communications Systems", IEEE Comm. Mag., pp. 56 69, Sept. 1998

[3] W. M. DeSevilla and E. Sousa, "Fading Resistant Transmission from Several Antennas", Proc. IEEE PIMRC'95, pp. 1218-1222, 1995.

[4] T. Heikkinen and A. Hottinen, "On Downlink Power Control and Capacity with Multi-Antenna Transmission", Proc. Conf. Inf. Sci. and Syst. (CISS'98), Princeton, pp. 475 - 479, March 1998.

[5] A. Hottinen and R. Wichman, "Transmit Diversity by Antenna Selection in CDMA Downlink", Proc. of IEEE $5^{\text {th }}$ Int. Symp. on Spread Spec. Tech. and Appl., pp 767 - 770, Sept 1998.

[6] T. Eyceoz, A. Duel-Hallen, and H. Hallen, "Deterministic Channel Modeling and Long Range Prediction of Fast Fading Mobile radio Channels", IEEE Comm. Letters, Vol. 2, No. 9, pp. 254 - 256, Sept. 1998.

[7] T. Eyceoz, S. Hu, and A. Duel-Hallen, "Performance Analysis of Long Range Prediction for Fast Fading Channels", Proc. of CISS'99.

[8] S. Hu, H. Hallen and A. Duel-Hallen, "Physical Channel Modeling, Adaptive Prediction and Transmitter Diversity for Flat Fading Mobile Channels," Proceedings of SPAWC'99.

[9] S. Hu, H. Hallen and A. Duel-Hallen, "Adaptive Power Control Using Long Range Predictiopn for Realistic Fast Fading Channel Models and Measured Data", to appear in Proceedings of ISCTA'99

[10] J. G. Proakis, Digital Communications, McGraw-Hill, New York,1995.

[11] W. C. Jakes, Microwave Mobile Communications, IEEE Press, 1993.

[12] A. J. Goldsmith and S. G. Chua, "Adaptive Coded Modulation for Fading Channels", IEEE Trans. on Comm., vol. 46, No. 5, pp. 595 - 601, May 1998.

[13] D. L. Goeckel, "Adaptive Coding for Fading Channels using Outdated Channel Estimates", Proceedings of VTC, May 1998.

[14] E. Biglieri, G. Cairo, and G. Taricco, "Coding and Modulation under Power Constraints", IEEE Personal Communications, pp. 32-39, June 1998.

[15] G. L. Stuber, Principles of Mobile Communications, Kluwer, 1996 